\title{
VISIÓN HUMANISTA DE LA BIOÉTICA*: HOMENAJE A FRANCISCO LEÓN CORREA
}

Ronald Cárdenas Krenz **

\section{HUMANISTIC PERSPECTIVE OF BIOETHICS': IN HONOUR OF FRANCISCO LEON CORREA}

\section{INTRODUCCIÓN}

Es muy honroso para mí el poder presentar ante nuestro claustro universitario, para su reconocimiento como Profesor Extraordinario, en calidad de Honorario, a una persona de los méritos y valía del distinguido bioeticista español Dr. Francisco León Correa ${ }^{2}$

Nuestra Universidad, vinculada siempre con la defensa de la vida, está naturalmente comprometida con la bioética, tarea en la que ha venido trabajando desde hacer varios años con reconocidos bioeticistas como los doctores Roberto Llanos o Patrick Wagner.

\footnotetext{
* Discarso de presentación del Dr. Francisco León Conrea como profesor Extraordinario en la Calidad de Honorario de la UNIFE, realizado et 1.8 de septiembre de 2012 .

** * Decano (a.t.) de la Facultad de Derecho de la UNIFE. Ptofesor de las Facultades de Derecho de la UNiFE, Lima y ESAN. Ex Superintendente de la SUNARP, Presidente del Consejo del Notariado y Presidente del Conscjo Nacional de Catastro. Socio de CDOM Consukores. Experto en temas hiberales de la fundacion alemana f'riedrich Naumann. Incluidoent la lista de Who's who in Ethics de la UNESCO. Autor de diversas publicaciones.
}

1

Dr. Francisco León Correa's Lecture on his incorporation as licnorary professor extraordinarie of Universidad Femenina del Sagrado Corazón - UNIFE, realizad on septembre $18^{4 *}$.

Recepción: 20-09-2012

Aceptación: 25-09-2012

2

Franciscu Javier Leon Correa es Doctor en Filosofía y Letras, por la Universidad te Valfadolid, España (1985). Especializado en las corritutes conternoráneas de f́tica y Antropología. Dédicado desde 1989 a la docencia e investigasiun en bioćlica. Ha cjercido la dontncia en las universidades de Teón, Valladolid y Santiago do Compostela en España (1997-2001), como Lambién en las universidades Católica, Diego Portales y Andrés belio en Chile (des de el 2002 en adelante). Actualmente, es Profesor Adjunto del Centro de Bioética de la Facultad de Medicina, de Ia Pontificia Unjversífad Católica de Chile. Ha sido uno de los furdadores y director de! Grupo de investigación er Bioética d. Galicia (1990-2001). promotor y director de la importarise revista Cuademes de Bìútica. Miemebro fundador de la Asociación Española de Bioctica y Etíca Médica (AEBl), secrefario desde cncro de 1995 a 2000 . Es I'residente de la Federación Latinomericana y del Curibe de Institticiones de Bicética (FE.LAJBE) y Presidente de la Sociedact Chilena de Biostica además de Secretaxio de la Sociedad Chilena de Drrecho Santitario. También es colaborador del Frograrara dr. Bioćtica OPS/OMS. Autor de diversos libros y duccras de artículos publicados en revistas especializadas de Europa y Latinoamérica. Dentro de sus jineas de especialización, pueden destacarse las de Ética clúnica, bioética, salud pública y politicas de salud, y justiciásocial ex salua. 
En la Facultad de Derecho, en abril de 1998, organizamos el primer Seminario Internacional sobre Bioética y Biojurídica y, luego. en noviembre de 1999, bajo el promotor empuje de la Dra. Gabriela Arant́bar Fernández Dávila -ex Presidenta de la Comisión Interamericana de Mujeres y Decana de la Facultad y con el concurso activo de la recordada filósofa y jurista española, Dra. María Dolores Vila-Coro,-Presidenta de la Sociedad Española de Biojurídica y Bioética-, creamos una Cátedra de Bioética que se convirtió en la primera Cátedra UNESCO de Bioética y Biojurídica en el mundo, y en cuyo desarrollo trabajamos durante 9 años.

Concluido el convenio, seguimos trabajando en ella como Cátedra UNIFE de Bioética y Biojurídica, siendo la primera Facultad de Derecho en el Perú y la primera Maestría en Derecho, en tener una cátedra específica sobre la materia.

Cabe también señalar que en la bibliografía nacional solo hay dos libros publicados por una Facultad de Derecho que se han ocupado específicamente de la Bioética: Uno de ellos "B. vética y Biojurídica: La Unidad de la Vida", publicado en el año 2002 y "Temas de Bioética y Derecho", publicado en el año 2007, ambos editados por la UNIFE.

Por todo ello y por los principios y valores que inspiran a la Universidad, es particularmente grato para nosotros el poder enriquecer nuestra Cátedra con la presencia del Dr. Francisco León Correa, con quien no solo compartimos temas comunes, sino, sobre todo, principios y valores, como puede corroborarse fácilmente al repasar algunas de las ideas fundamentales de su Iúcido pensamiento, el cual parte de una bioética personalista, cuyas bases reposan en la dignidad de la persona, incluyendo, como señala José Miguel Serrano, una apertura a la ética de las virtudes, el enfoque deontológico y determinadas fórmulas aristotélicas.

\section{Fundamentación y concepto de la bioética en el pensamiento de Francisco León}

Su producción bibliográfica, su amplia trayectoria docente, su aporte como creador en Santiago de Compostela de la más importante revista española sobre Bioética en español, llamada "Cuadernos de Bioética", ofreciendo un invalorable aporte para una alternativa a la bioética dominante en España, constituyen de por sí destacados logros, mas a ello debe agregarse su permanente inquietud por la bioética en Iberoamérica, donde radica desde hace algunos años. De allí que no sea casual que haya sido elegido presidente 
de la Sociedad Chilena de Bioética, como también Presidente de la Federación Latinoamericana de Instíuciones de Bioética, además de ser -por lo menos hasta donde conocemos- el más importante recopilador de bibliografía hispanoamericana sobre la materia.

$\mathrm{AI}$ analizar los fundamentos éticos de la Bioética, Francisco León plantea que existen cuatro líneas que confluyen en el nacimiento de la Bioética como ética aplicada: "los inicios de una ética ecológica, los problemas de la investiģación con seres humanos, los progresos científicos y técnicos de la medicina moderna y los cambios en la atención de salud con el nacimiento de los sistemas más o menos universales de seguro social ${ }^{3 / \prime}$.

La Bioética supone entonces un intento de lograr un enfoque secular, interdisciplinario, prospectivo, global y sistemático, de todas las cuestiones éticas que conciernen a la investigación respecto al ser humano $y$, especialmente, a la biología, la medicina y la atención en salud ${ }^{4}$.

\begin{abstract}
Ahora bien, su visión de la bioética corresponde a una línea personalista ${ }^{5}$, que puede definirse como "la búsqueda del conjunto de exigencias del respeto y de la promoción de la vida humana y de la persona en el sector biomédico y la atención en salud $d^{6 \prime}$, siendo de notar que esta perspectiva no está limitada a un pensamiento cerrado, sino más bien abierto al diálogo con otras vertientes bioeticistas, apertura que distingue y enaltece su pensamiento.
\end{abstract}

Siendo una perspectiva humanista, no plantea una ética individualista que prescinda de las repercusiones sociales de su quehacer, en busca de una

3 León, Francisco. La pronoción de la dignidad de la persona en el ámbito biomédico. Madrid, Ediciones Palabra, $201 \mathrm{~L}, \mathrm{p}$. 13-I4.

4

íbid., p. 16- 17. De la cíada definición, pueden desprenderse las catacterísticas propias de la Bioética: La interdisciplinariedad, el debate ético en una sociedad plural basado en la tolerancia, la elaboración de unia teoría de fundamentación apoyada en unos principios claros y concretos (no maleficencia, justicta, atutonomía y beneficencia) y la aplicación efectiva de esos principios en el ámbito clínico, con dos aspertos importantes: Una metodología especial de análisis clínicos y la creación de comités de ética asistencial o ética clínica en los hospitales, que han difundidoel interés por la Bioética y que son un instrumento "institucionalizado" al servicio de la calidad de atención en salud y de la humanización de la relación clínica. (p. 17-18).

5 Resal ta Francisco León que la aportación que des de el personalismo se hace al debate biótico es poner en el centro de la discusión a la persona concreta, destacando que dicha corriente "surge frente a la vision individualista y racionalista del hombre, porque al considetarlo como persona lo entiende más que como pura razón individual. La persona es razb́n, voluntãd y corazón". ("Fundamentos y principios de bioética clínica, institucional y social". En Acta Bioétiça 2009; 15(1), p. 72).

${ }^{6}$ León, Francisco. La promoción de la dignidad de la persona en el ámbito biomédico. Op. Cit., p. 20. 
autoperfección pexsonal; sostiene más bien que "el hombre es capaz de actuar" éticamente sólo desdo la libertad" ${ }^{\prime \prime}$, mas no una libertad absoluta, sino de una libertad que se ejerce y se enriquece con el respeto de las demás y que se ejerce con aignidad, mostrando que es falsa la opsición entre libertad y dignidad, afimando que: "es fundamental en el ámbito de la bioética, entender el valor complementario que tienen la dignudad y la libertad del tombre, sin contraponerlos en falsas disyuntivas. ${ }^{\text {" }}$

Empero, si bien reconoce la autonomía como expresion de la dignidad de la persona humana, y que hos principios fundamentaíes del respeto la vida humana y de autodeterminación de lâ persona son complementarios, elio no le impide detenerse a analizar el problema que autores norteamericanos tratan de resolve: cómo equilibrar los principios de aztononía con los de justicia y beneficencia, en un sistema que ha privilegiado de modo unilateral la autonomía del paciente, que ha levado a una medicina defensiva contraria a los intereses de mécicos y pacientes, siendo necesario articular ahora dicho principio con una beneficencia (no paternalis a) y la idea de responsabilidad como principios ligados a elia.?

Por otro lado, resalta el papel de la familia, fundamental por ejemplo -y en especial en Latinoamérica- en las decisiones clínicas y en el consentimiento informado, oponiéndose así al indiviưualismo más extremo.

Para él, lo prinero en ética es «Desear eficazmente una vida lograda» y la ética es ese deseo, antes que una mera normativa, y la libertad es el medio para lograr la felicidad. ${ }^{10}$ Pero ¿qué es una vida plena, lograda, realizada?, nos dice que es "aquella en la que hay concordancia entre lo que acabamos haciendo y siendo, y los ideales que nos marcamos desde nuestras potencialidades, incluyendo las personales y las sociales, exigibles desde la justicia para todos".

\footnotetext{
7 I.eon Correa, Francisco. "Dignidad Humana y Derechos Humanos en Bioética". En: URBE et iUS: Newsletter Nro, 20, p. 10.

8 León Correa, Francisco. "Dignidad Humanay Detechos Flumanos en Bioética". Op. cit., f.6.

9 León Correa, Francisco. "Autotomía y beneficencia en la ética clitrica: ni paternalismo ni medicira défensiva". Op. cit., p. 258.

10

León Corrca, Francisco. "Eil aborto desde la bioćlica: ¿Autonorúa de la mujer y del médico?". En: Cladernos de Bioélica. XX1, 2010/1ㅜ, p. 89-90.

11

León Correa, Francisco. "Fundamentes y prirxcipios de bioética clínica, institucional y social". Op.cil., p.73.
} 
Ahora bien, como Bioética y Derecho buscan el mismo fin: la promoción del respeto a la vida humana y a los derechos fundamentales, entonces ambos deben andar unidos.

\section{Acerca del aborio y la autoromia de la persona}

Analizando el aborio desde la perspectiva del principio de autonomía, observa que la cuestión del aborto no es solo un tema de la autonomía de la madre; hace referencia al choque entre dicha autonomía y la beneficencia debida al feto como ser humano, cuya dignidad debe respetarse.

Señala, coincidiendo con Gonzalo Herranz, que hoy, la discusión sobre el aborto ya no es la clásica de si es un ser humano o no. Su centro de gravedad se ha desplazado a la cuestión de un pretendido derecho de abortar de la madre, en virtud de su autonomía moral, independientemente de los da os de la embriología y la reflexión antropológica. ${ }^{12}$

Partiendo de que el hombre es el único ser verdaderamente líbre, profundamente libre, considera que su libertad se manifiesta como poder. Mas la idea de una libertad absoluta llevaria a la negación de la propia libertad. La autonomía no es ilimitada, es parte de la libertad responsable del ser humano que sabe que debe actuar respetando la libertad de los demás, y sabe también que debe actuar conforme a su propia dignidad de persona. Esto le obliga moralmente a cuidar y respetar los bienes básicos que conforman su dignidad: la vida, la salud, la conciencia propia y de los demás, dice Francisco León citando a A. Gómez-Lobo. ${ }^{13}$

Tanto la mujer, como el feto, son seres humanos dignos, reclama, y la dignidad no se puede medir matemáticamente, advirtiendo que la «solución rápida» del aborto nunca resuelve nada por sí soĺa, sino que empeora la marginación de la mujer.

Por eso, el aborto no es solo un daño al feto, sino que la madre también se provoca a sí misma una pérdida irreparable en su propia dignidad tnoral

\footnotetext{
12 León Correa, Francisco. "El aborto des dela la biótica: ¿Autonomía de la mujer y del médico?". Op. cit., p. 88,

13 Ibid., p. 84.
} 
como persona. De allí que sea necesario encontrar soluciones que respeten la dignidad de todas las personas involucradas, incluyendo al hijo no nacido.

En el caso de Ia vida humana en estado embrionario o fetal -destaca con acierto-, no se puede confundir la potencia de ser con el no ser. El embrión. tiene ya la cualidad humana, ya es un ser humano. ${ }^{14}$

Adicionalmente -y ésta es otra clave fundamental en el pensamiento de Francisco León-, la persona humana no es valiosa por lo que «tiene», sino por lo que "es» ${ }^{15}$; recordando que es necesario tener en cuenta que no todo lo permitido legalmente es éticamente correcto.

Todos estamos convocados a vivir nuestra vida con una autonomía desde la que nos hacemos cargo de nosotros mismos, con responsabilidad. Ahora bien, nuestra realización no puede ser individualista, la apertura a los otros es precisamente lo que nos construye moralmente y posibilita la realización personal. ${ }^{16}$ En tal virtud, debemos asegurar los derechos de las más vulnerables, la mujer y el concebido, sin olvidarse de los deberes de todos para con ellos.

\section{Diálogo Bioética y Religión}

Sobre este tema, tan sensible y relevante en nuestro tiempo, León coincide en el planteamiento de un điálogo fecundo entre Bioética y Religión, viendo la bioética como un diálogo plural entre diversas ciencias y corrientes de pensamiento sobre los aspectos éticos de la atención en salud y el cuidado de la vida humana en el ámbito biomédico, apuntando que "el diálogo es posible si, por un lado, se reconoce el aporte de la ética cristiana a la bioética y, por otro, se respeta la diversidad de racionalidades y cosmovisiones presentes en la sociedad". ${ }^{17}$

\footnotetext{
14 León Correa, Francisco. “El aborto descle la bioética: ¿Autonomía de la mujer y dęl médico?". Op. cit., p.86.

15 León Correa, Francisco. "Digridad Humana y Derechos Humanos en Bióstica", Op. cit. p. 9.

16 León Correa, Francisco. "El aborto desde la biótiça: ¿Autoriomía de la mujer y del médico?". Op. cit., p. 90.

17 Ver para más detalle, el artículo de Francisco León Correa. "Bínética y religión cristiano-católica: Dos racionalidades complementarias", publicado en Acta Bioética 2010; 16 (1); p. 9-10.
} 
En uno de sus últimos libros "Ternas de Bioética Social", señala que se ha reiniciado defintivamente el díálogo -y a ello ha aportado también el desarrollo de la bioética -entre fe y razón, entre religión y ciencia, más allá de los extremismos propios de la sociedad postmoderna y secularista del siglo $X X, y$ de las posiciones intolerantes, más bien ideológicas que religiosas. En la confrontación se pierden de vista las razones del otro y se intenta construir un mundo a la medida de nuestras personales razones excluyentes. La historia del cristianismo es la historia de un diálogo fructífero entre fe y razón, superador de ideologías irracionales. En el diálogo, ai entender y respetar -aunque no se compartan- las razones del otro, entenderemos mejor nuestras propias ideas, y podremos ofrecer entre todos soluciones positivas in un mundo necesitado de clarificación y profundización en los valores morales". ${ }^{18}$

Citando a Javier Gafo ${ }^{19}$-a quien también tuvimos el honor de tenerlo en nuestra Universidad hace varios años-, menciona valores cristianos de especial relieve en bioética, como el valor y la intrínseca dignidad de todo ser humano, por encima de las circunstancias externas y personales; la vida humana como valor fundamental del que no se puede disponer arbitrariamente; la ética de Jesús como ética en la libertad; la ética evangélica basada en el amor, en el don gratuito, en el dar sin esperar.

Lo interesante -resalta- es que "Todos estos valores, genuinamente evangélicos, son asumibles desde una ética no-creyente. Permiter un diálogo no sólo interdisciplinar, sino también entre distintas cosmovisiones y concepciones de la vida".

En esa perspectiva, suscribe la necesidad de buscar una responsabilidad solidaria efectiva y universalistamente abierta, más allá de los intereses de un 'nosotros' restrictivo.

18 León Correa, Francisco Javier. Temas de Bioética Social. Santiago de Chile, Fundación Inierameriçana Ciencia y Vida, 2011 , p. 181 .

19 Ver para más áetalle, el articulo de Francisco León Correa. "Biótica y religión cristiano-católica: Dos racionalidades complementarias". Op. cit., p. 12. 


\section{Tratamiento del paciente}

Ese espíritu solidario y humanista se expresa en sus consideraciones acerca del tratamiento de los pacientes, siendo aquí interesante la propuesta que nos hace de legislar sobre sus derechos atendiendo al modelo imperante en cada país. No es deseable mantener el obsoleto modelo paternalista, pero tampoco se trata de importar modelos sin más. ${ }^{20}$

Frente a la autonomía radical planteada especialmente por autores anglosajones, que siguen por ejemplo a Stuart Mill, ${ }^{21}$ observa con honestidad que, en la práctica, nos enfrentamos muchas veces a pacientes no competentes o con la competencia disminuida; ello aparte de la importancia en el ámbito latinoamericano, del papel de la familia en este tipo de decisiones, ya resaltada anteriormente. ${ }^{22}$

Ahora bien, un alcance importante que hace es mencionar que "La autonomía no es sólo el respeto por las decisiones librea, voluntarias e informada del paciente, a través del proceso del consentimiento informado, sino también el deber ético de los profesionales de promover la competencia y de ayudar -sin paternalismos-a que el paciente pueda ejercer una libertad responsable ${ }^{\prime 2}{ }^{23}$

20 . 257

21 Dice Stuart Minl en su libro Sobre la liberlad (Madrid, Aliazza Editorial, 1970: “Ningún hombre puede, en buena lid, scr obligado a actuar o a tbstenerse de hacerto. porque de esa actuación o abstención haya de derivarse un bien para él, porạue ello le ha de hacer más dichoso, o porque, en opinión de los demás, hacerlo sea prudente o juslo. Éstas son buenas razones para discutir con él, para convencerle o para suplicarle, pero no para obligarle o causarle daño alguno si obra de thodo diferente a nuestros deseos. Para que esta coacción fuese justificable, sería necesario que la contiucta dic cstc hombre tuviese pur objeto el perjuicio de otro. Pata aquello que no le atañe más que a el, su independencia es, de hecho, absoluta. Sobre sí mismo, sobre su cuerpo y su cspíritu, el individuo es soberano".

22

"Pero en la práctica nos enfrentamos muckas vects a pacientes no competertes o con la competencia disminuida, que hacen diffícil la aplicación inmediata del principio de Stuart Mill, aunque estuviéramos de acuerdo con el, de forma que debemos ir a decisiones subrogadas, cada vez más alejactas de esa "soberanía individual". Tambiéth en el âmbito latinoamericano, la familia cumple un papel importante en las decisiones clinicas y en el consentimiento informado, frente al individualismo nás extremo. $Y$ asimismo, frente a esa independencia del paciente que decide, muchas veces vemos situaciones de desigualdad de recursos, injusticias en el acceso igualitario a los ruidados de salud, grandes diferencias entre la medicina pública y la privada, y fuertes desigualdades ente pacientes muty bien informados y pacientes con una muy baja educación sanifaria". León Correa, Frarkisco. "Autonomía y beneficencia en la ética clínica: ni paternalismo ni medicina defensiva". Op. cit., p. 257.

23

León Correa, Francisco. "Fundamentos y principios de bióstica clínica, instituciontal y social". En: Acta Biólica $2009 ; 15$ (1). p. 76 . 
Resalta la importancia de tener una perspectiva relacional, que considere siempre al otro como sujeto, con estima de sí, con autonomía. Esa apertura a los otros es lo que nos construye moralmente y posibilita la realizición personal, lo cual es algo que deben tener en cuenta tanto médico como paciente.

En su libro Fundamentos y Principios de Bioética Clinica, Institucional y Social, fundamenta la necesidad de ampliar el campo de la bioética en Latinoamérica de una ética clínica a una bioética social, para abordar los dilemas éticos institucionales, de salud pública, políticas y reformas de los sistemas y legislación. Propone entonces incorporar al debate académico en bic ética estos aspectos de su dimensión institucional y social; y, de otro lado, "desarrollar el sistema de la bioética de principios, fundamentando desde el personalismo y la ética dialógica los preceptos complementarios que puedan ser utilizados en las resoluciones de casos y toma de decisiones". ${ }^{24}$

\section{Sobre la Dignidad humana y el contenido de la Bioética}

Considera que "el respeto a la dignidad de la persona humana es el tema central en los actuales debates de Bioética, y en general de toda ética social contemporánea. Es la base común sobre la que podemos construir un consenso ético general entre todos, en nuestras sociedades plurales y multiculturales, con diversidad de posiciones éticas". 25

La Bioética -sostiene- no se debe restringir a un ejercicio de toma de decisiones, o en la implementación de modelos éticos que ayuden en las decisiones clínicas. Debe ir más allá, y profundizar en su fundamentación, interrogándose por los actores presentes en la investigación biomédica y en la atención clínica.

Por ello, tenemos que saber cómo debemos tratar a las personas, y cómo debemos comportarnos las personas, a partir del reconocimiento de nuestra dignidad. Comprender la dignidad y la libertad como valores no divergentes sino complementarios. Saber que el ser personaí, trasciende la dimensión puramente biológica desde su libertad.

\footnotetext{
24 Anota Francisco León que la aportación clave que desde el personalismo sc hace al debate bioético es "poner en ei centro de la discusión un "cable a tierra", centrar todo en la persona concreta, del médico o del profesional de la salud, del phaciente y del usuario". León, Francisco. La promociós de la äignidad de la persona en el ámbito bionédico. Op. cit., p. 34.
}

León Correa, Francisco. "Dignidad Humana y Dercchos Humanos en Bioética". Op. cit., p. I. 
En esa línea de pensamiento, expresa que: "Llamamos bioética a la búsqueda del conjunto de exigencias del respeto y de la promoción de la dignidad de la vida humana y de la persona en el sector bio-médico". Agregando desde ei personalismo el primado y la intangibilidad de la persona humana, considerada como valor supremo, punto de referencia, fin y no medio ${ }^{26}$, deduciendo que ninguna discusión sobre la "calidad de vida" puede prescindir de una voluntad de respeto por la vida misma. ${ }^{27}$

Reconoce que la ciencia y la técnica permiten una vida más digna al mejorar el conocimiento de la naturaleza y posibilitar un actuar más libre. "Pero el poder del conocimiento y de la ciencia no son el único ni el principal factor de libertad. Lo que permite a la libertad humana profundizar en su propio actuar y, por tanto, aumentar la dignidad de la persona, es la comunicación con otras libertades. Y no cualquier comunicación, sino la relación libre basada en el amor, en el deseo del bien. Donde hay necesidad no hay amor, sino sometimiento, porque no hay libertad. La necesidad nunca funda el amor" ${ }^{28}$

\section{Algunas ideas complementarias}

- El derecho no puede desentenderse u oponerse a la moral.

- La ética no puede definirse por consenso, pues respondería a una concepción utilitarista o pragmática.

- La ética tiene dos caras: Una que apunta al bien que debemos realizar y que llamamos felicidad, y la que nos muestra el deber o la norma que debemos cumplir, agregando luego que la ética se vive en plenitud cuando se articuian sus dos caras: la teleológica y la deontológica. ${ }^{29}$

- Debemos ir a una profundización en la enseñanza de la bioética y en el aprendizaje de la Bioética por parte de los profesionales sanitarios muy especialmente.

\footnotetext{
26 León Correa, Fitancisco. “Dignidad Humana y Derechos Fumanos en Bioética”, Op. cit., p.3.

27 Leon Correa, Frarkisco. Ibid., p.9.

28 León Correa, Francisco. Ibid., F, 8.

29 León Conrea, Francisco. "Fundanentos y principios de bioética clínica, institucional y sucia]". Op.cit., p.73.
} 
- La religión complementa a la filosofía, pues esta aporta lo que debemos hacer, mientras la religión aporta to oue debemos esperar. ${ }^{3.0}$

- El consentimiento informado no se limita a un acto aislado y puntual como puede ser la aceptación de una prueba o tratamierto, sino que se refiere "a algo más amplio, a un modo de entender la relación clínica como un diálogo ente agentes morales", el cual tiene tres piezas fundamentales: a) Lat capacidad o competencia de la persona para adoptar decisiones respontables; b) La voluntariedad o libertad de coacciones internas y externas; y la información sustantiva o necesaria para que el paciente pueda decidir. ${ }^{31}$

- No basta con una medicina a la defensiva que simplemente atiende y defiende los derechos del paciente, no basta con cumplir con la no maleficencia, por no hacer mala praxis, no basta con cumplir los deberes de justicia; no basta con esto para conseguir una humanización de la atención sanitaria. Hace falta recuperar el principio de benef́icencia médica, no paternalista, y a la vez una fundamentación de esos cuatro principios de la Bioética en una visión del paciente como persona, del médico como persona.

\section{¿Una Bioética Latina?}

Francisco León plantea la posibilidad de una Bioética Latina, sosteniendo que tenemos que partir de una Bioética más fundamentada en la dignidaa de la persona que en la exaltación del principio de atonomía del paciente; en un equilibrio entre esos principios, superando el viej̧o paternalismo médico, manteniendo esos valores que han existido siempre en la medicina Latinoamericana, al iguai que la española, de profundo humanismo de los médicos, de una relación de amistad y cercanḱa con los pacientes.

Sostiene que un reto ineludible para la Bioética en América Latina es dar una fundamentacion a las posibles y deseables respuestas a problemas pasados $y$ muy presentes de nuestros sistemas de salud, sin dejar de tener en cuenta el

30 Ver para más detalle, el articuh de Francjsco León Correa. "Biótica y religión cristiano-católica: Dus racionalidaedes complementarias". Op.cit., p. 14.

31 León, Framisco Javiet y Ma. Angeliea Piwonka. Información al paciente y conzsentimiento informado. Bioética y Enjémerín. Santiagro de Chile, Fundación Ciencia y viđa, 2011, p.8. 
contexto social, la política y hasta las creencias de la región, desde la perspectiva de una bioética social.

\section{Apuntes finales sobre un tema crucial: la eutanasia}

Francisco León no comparte la idea de distinguir una eutanasia pasiva de una activa, por considerarla equívoca, pues la eutanasia es toda acción u omisión que deliberadamente pretende acelerar u obtener la muerte del paciente sin dolor, por compasión o porque el paciente autónomamente lo solicita para que el profesional sanitario que lo atiende provoque su muerte.

Explica que aplicar métodos paliativos que pueden vencer cl dolor o hacer más humanos los últimos momentos del moribundo, no es eutanasia, aunque acorten en parte la vida del paciente. Anotando que no pertenece a la razón de ser de la medicina el intervenir para hacer daño.

La eutanasia es rechazada, prácticamente po. la totalidad de los médicos en Europa, salvo en Holanda, aunque ya hay preocupación por el deslizamiento y por el aumento de las eutanasias sir consentimiento previo del paciente.

Todo ello aparte del tema del oscurecimiento de conceptos, para generat una confusión ética y semántica, como cuando se hablar de "buena muerte" para referimos a la eutanasia, "selección neonatal" para referirse al infanticidio de acabar con la vida de niños malformados, "suspensión de nutrición y líquidos" para no decir norir de hambrey sed

Anota que en ensañamiento terapéutico es también una falta deontológica y que la eutanasia socava la confianza que debe presidir la relación médico/paciente, en la que es esencial el convencimiento de que el médico no abandonará nunca a su enfermo ni nunca le infligirá ningún daño deliberado; ello aparte de que se relaj̧e la actitud de dedicación sacrificada del médico como cuidador de vida.

Por todo lo expuesto, solicito a usted, Hna. Rectora, tenga a bien imponer al señor Doctor Francisco León Correa, la distinción correspondiente y otorgar el Diploma que lo acredite como Profesor Honorario de la Universidad Femenina del Sagrado Corazón. 


\section{REFERENCIAS}

León Correa, Francisco. "Autonomía y beneficencia en la ética clíni va: ni patemalismo ni medicina defensiva". En Revista Biomedicina (on line). Disponible en internet http://www.um.edu.uy/docs/ revistabiomedicina/2-3/autonomia.pdf, consultado el 02 de septiembre de 2006.

León Correa, Francisco. "Bioética y religión cristiano-católica: Dos racionalidades complementarias", En: Acta Bioética (on line) Disponible en Internet http:/actabioethica.cl/docs/acta21.pdf, consultado el $\mathrm{C} 2$ de septiembre de 2012 .

León Correa, Francisco. "Dignidad Humana y Derechos Humanos en Bioética". En: URBE et IUS: Newsletter Nro. 20 (on line). Disponible en Internet ht p:/ / www.urbeetius.org/newsletters/ 20/news20_correa.pdf, consultado el 02 de septiembre de 2012.

León Correa, Francisco. "El aborto desde la bioética: ¿Autonomía de la mujer y del médico?". En: Cuadernos de Bioética. XXI, 2010/1 ${ }^{\text {a }}$ (on line). Disponible en Internet http://www.aebioetica.org/rtf/06BIOETICA-71.pdf, consultado el 03 de septiembre de 2012.

León Correa, Francisco. "Fundamentos y principios de bioética clinica, institucional y social". En: Acta Bioética 2009 (on line) Disponible en Internet http://www.actabioethica.cl/docs/acta19.pdf, consultado el 03 de septiembre de 2012 .

León, Francisco Javier y Ma. Angélica Piwonka. Información al paciente y consentimiento informado. Bioética y Enfermería. Santiago de Chile: Fundación. Ciencia y Vida, 2011.

León, Francisco. La promoción de la digniada de la persona en el ámbito biomédico. Madrid: Ediciones Palabra, 2011.

León Correa, Francisco Javier. Temas de Bioética Social. Santiago de Chile: Fundación Interamericana Ciencia y Vida, 2011, p. 181. 
